\title{
INFLUÊNCIA DE VÓRTICES DE MESOESCALA NA CONCENTRAÇÃO DE CLOROFILA DA CONFLUÊNCIA BRASIL-MALVINAS: MECANISMOS INFERIDOS POR SENSORIAMENTO REMOTO
}

\author{
ANGEL-BENAVIDES, I.M. ${ }^{1 A *}$; PILO, G.S. ${ }^{18} ;$ DIAS, F.B. ${ }^{1 B}$ \& GARCIA, C.A.E. ${ }^{1 C}$ \\ 1. Laboratório de Estudos dos Oceanos e Clima, Instituto de Oceanografia, Universidade Federal do \\ Rio Grande (FURG), Rio Grande, RG, Brasil \\ A. atualmente em: Institute of Coastal Research, Helmholtz-Zentrum Geesthacth, Geesthacht, \\ Alemanha. \\ B. atualmente em: Institute for Marine and Antarctic Studies, University of Tasmania, and CSIRO \\ Oceans \& Atmosphere Flagship, Australia. \\ C. atualmente em: Departamento de Geociências, Centro de Filosofia e Ciências Humanas, \\ Universidade Federal de Santa Catarina, Florianópolis, SC, Brasil. \\ ${ }^{*}$ Corresponding author: ingrid.angel@hzg.de.
}

\begin{abstract}
Angel-Benavides, I.M.; Pilo, G.S.; Dias, F.B. \& Garcia, C.A.E., (2016). Influência de vórtices de mesoescala na concentração de clorofila da confluência Brasil-Malvinas: mecanismos inferidos por sensoriamento remoto. Braz. J. Aquat. Sci. Technol. 20(1). elSSN 1983-9057. DOI: 10.14210/bjast.v20n1. This study aims to (a) identify spatial patterns in surface phytoplankton abundance associated with mesoscale eddies of the Brazil-Malvinas Confluence (BMC), and (b) infer the responsible physical mechanisms for such patterns. We here select 108 large eddies from a global eddy census database and test four eddy mechanisms that may affect surface chlorophyll-a concentration [chla]: (a) eddy-pumping, (b) eddy Ekman pumping, (c) eddy gradient advection, and (d) horizontal fluid advection. We calculate correlations between [chla] spatial distribution and physical characteristics associated with each of the four mechanisms. We find that eddy pumping is the dominant mechanism responsible for the [chla] spatial pattern as the anomalies associated with eddy cores have a significant correlation with eddy amplitudes. In addition, the high mean non-linear parameter (3.41 for cyclones and 2.83 for anticyclones) suggest the horizontal fluid advection mechanism is present in BMC eddies. Therefore the non-linear advection of fluid can also be important in maintaining the eddy-pumping signature. The eddy Ekman pumping is evident only for cyclonic eddies and, compared to the eddy-pumping mechanism, had a weaker influence in the observed [chla] patterns. The signature of the eddy gradient advection mechanism is manifested only in anticyclonic eddies traveling along meridional [chla] gradients. The effectiveness of this mechanism shows itself to be more dependent on the eddy rotation speed than on the background [chla] gradients.
\end{abstract}

Keywords: Mesoscale eddies, Brazil-Malvinas Confluence, Remote sensing, Ocean color.

\section{INTRODUÇÃO}

Vórtices oceânicos são feições de mesoescala definidas por linhas de corrente fechadas, podendo ser ciclônicos (rotacionando no sentido horário no hemisfério sul) ou anticiclônicos (rotacionando no sentido oposto no mesmo hemisfério). O sentido de rotação de um vórtice afeta o comportamento da superfície livre no interior da feição, resultando em anomalias negativas (positivas) da altura da superfície do mar para vórtices ciclônicos (anticiclônicos).

Mecanismos físicos associados aos vórtices podem afetar os padrões espaciais da concentração de clorofila ([chla]), proxy da abundância de biomassa fitoplanctônica superficial. Esses mecanismos são: (i) bombeamento vortical (eddy-pumping), (ii) bombeamento vortical de Ekman (eddy-Ekman pumping), (iii) advecção vortical de gradientes (eddy advection), e (iv) advecção horizontal de fluído. A Figura 1 ilustra os três primeiros mecanismos, adaptada de Siegel et al. (2011) para o Hemisfério Sul.

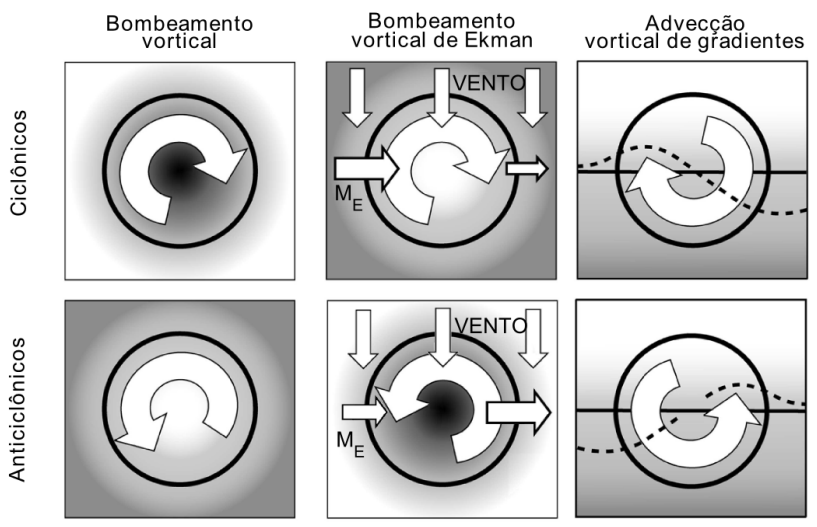

Figura 1 - Representação de três dos mecanismos através dos quais os vórtices de mesoescala alteram os padrões espaciais das propriedades bio-óticas, como a [chla]. A cor de fundo indica como a [chla] varia, onde maiores (menores) concentrações são representadas por cores mais escuras (claras). O transporte de Ekman é representado pelas setas rotuladas como ME. Adaptado de [Siegel et al., 2011] para o hemisfério sul e gradiente meridional de [chla] aumentando para sul. 
O bombeamento vortical (Figura 1, esquerda) causa o deslocamento das isopicnais em direção à superfície, em vórtices ciclônicos, ou ao fundo, em vórtices anticiclônicos. Esse deslocamento resulta das anomalias na superfície livre do mar em resposta ao sentido de rotação dos vórtices. Em vórtices ciclônicos, o bombeamento vortical provoca ressurgência de águas com maiores concentrações de nutrientes no centro das feições (McGillicuddy \& Robinson, 1997) ou de células fitoplanctônicas concentradas em subsuperfície (Arístegui et al., 1997). Em vórtices anticiclônicos, o bombeamento vortical resulta em subsidência da camada de mistura (Tilburg et al., 2002), inibindo o crescimento fitoplanctônico (Eden \& Dietze, 2009). Dessa maneira, o bombeamento vortical resulta em maior (menor) [chla] no centro dos vórtices ciclônicos (anticiclônicos).

O bombeamento vortical de Ekman (Figura 1, centro) causa a convergência de àguas no interior de vórtices ciclônicos, ou a divergência de águas no interior de vórtices anticiclônicos. Esse bombeamento resulta do transporte de Ekman diferenciado nos hemisférios da feição (Martin \& Richards, 2001; McGillicuddy et al., 2007). Considerando um vórtice cuja velocidade rotacional tem magnitude constante, mas sentido contrário em hemisférios opostos da feição, sob o efeito de um vento espacialmente homogêneo, o estresse do vento será maior (menor) naquele hemisfério no qual o sentido da velocidade da água é oposto (igual) ao da velocidade do vento. Em vórtices anticiclônicos (ciclônicos), esta assimetria espacial do estresse do vento resulta em divergência (convergência) do transporte de Ekman no interior da feição e, portanto, em velocidades verticais positivas (negativas) no interior do vórtice, independentemente do sentido do vento. Dessa maneira, o bombeamento vortical de Ekman resulta em menor (maior) [chla], no centro dos vórtices ciclônicos (anticiclônicos).

A advecção vortical de gradientes (Figura 1, direita) causa a distorção de gradientes horizontais de [chla] de uma região, devido à ação da velocidade rotacional dos vórtices (Siegel et al., 2008; Chelton et al., 2011a). Considerando um vórtice ciclônico no hemisfério sul que se propaga em uma região onde a [chla] apresenta um gradiente meridional aumentando para sul, a rotação no sentido horário advecta para norte (sul) águas com maior (menor) [chla] no lado oeste (leste) da feição. A distribuição espacial de tal padrão de anomalias depende do sentido do giro do vórtice e do gradiente espacial da propriedade. Dessa maneira, a advecção vortical de gradientes resulta em um dipolo de anomalias de [chla] positivas e negativas (Chelton et al., 2011a).

A advecção horizontal de fluido realiza o transporte de massas de água entre o local de formação dos vórtices e sua trajetória (Robinson, 1983). Um indicador dessa advecção é a razão entre as velocidades rotacional e de propagação do vórtice, conhecida como parâmetro de não-linearidade dado por $\mathrm{U} / \mathrm{c}$, sendo $\mathrm{U}$ a velocidade rotacional do vórtice e $\mathrm{c}$ a sua velocidade de propagação (Chelton et al., 2011a). Um vórtice cujo parâmetro de não-linearidade é maior do que 1 apresenta um comportamento não-linear, mantendo uma estrutura coerente ao longo do seu deslocamento (Flierl, 1981) e sendo capaz de isolar e transportar água do seu local de formação.

A geração de vórtices de mesoescala na região da Confluência Brasil-Malvinas (CBM) tem sido observada em imagens da cor do oceano (Garcia et al., 2004), temperatura superficial do mar (Souza et al., 2006) e altura da superfície do mar (Lentini et al., 2006). A CBM apresenta maior [chla] quando comparada à Corrente do Brasil $(\mathrm{CB})$ e à Corrente das Malvinas (Brandini et al., 2000). A CBM é caracterizada pela mistura de águas subantárticas, frias e ricas em nutrientes, com águas subtropicais, quentes e pobres em nutrientes., Essa mistura foi sugerida por Brandini et al. (2000) como uma possível causa da ocorrência do máximo local de [chla], pois provê condições ambientais apropriadas (estabilidade e nutrientes) para um maior crescimento fitoplanctônico. Na CBM, vórtices ciclônicos apresentam [chla] média maior que a apresentada por vórtices anticlônicos (Saraceno \& Provost, 2012), como esperado pelo mecanismo clássico de bombeamento vortical. No entanto, as relações entre os padrões espaciais na [chla] associados aos vórtices e ao bombeamento vortical de Ekman, a advecção vortical de gradiente e a advecção horizontal de fluído ainda não foram avaliadas. $O$ presente estudo tem como objetivos: (a) qualificar as assinaturas espaciais na [chla] associadas à presença de vórtices na região da CBM e (b) inferir os mecanismos físicos responsáveis por tais assinaturas.

\section{MATERIAL E MÉTODOS}

\section{Banco de dados de vórtices}

A segunda versão do banco de dados de trajetórias de vórtices elaborado por Dudley B. Chelton e Michael G. Schlax (Chelton et al., 2011b) foi utilizada para a seleção dos vórtices analisados. A base de dados foi construída a partir de dados semanais de anomalia do nível do mar (Sea Level Anomaly - SLA) da série de referência da AVISO entre Outubro de 1992 e Janeiro de 2011, elaborada a partir de dados unificados de dois altímetros. O banco apresenta valores de deslocamento da superfície livre do mar em relação ao estado médio (amplitude do vórtice), velocidade rotacional, raio, sentido de rotação, data 
e coordenadas geográficas dos vórtices em cada momento de sua trajetória. Neste trabalho foram selecionados vórtices formados na região de alta energia cinética turbulenta associada à CBM, localizada entre $30^{\circ}-50^{\circ} \mathrm{S}$ e $30^{\circ}-60^{\circ} \mathrm{W}$ (Figura $2 \mathrm{~b}$ ), com raios médios extremos (superiores à mediana do raio médio dos vórtices da área) no período de 2003 a 2010. Somente foram incluídos vórtices com tempo de vida superior a 16 semanas, como sugerido por Chelton et al. (2011b), a fim de garantir a robustez dos resultados.
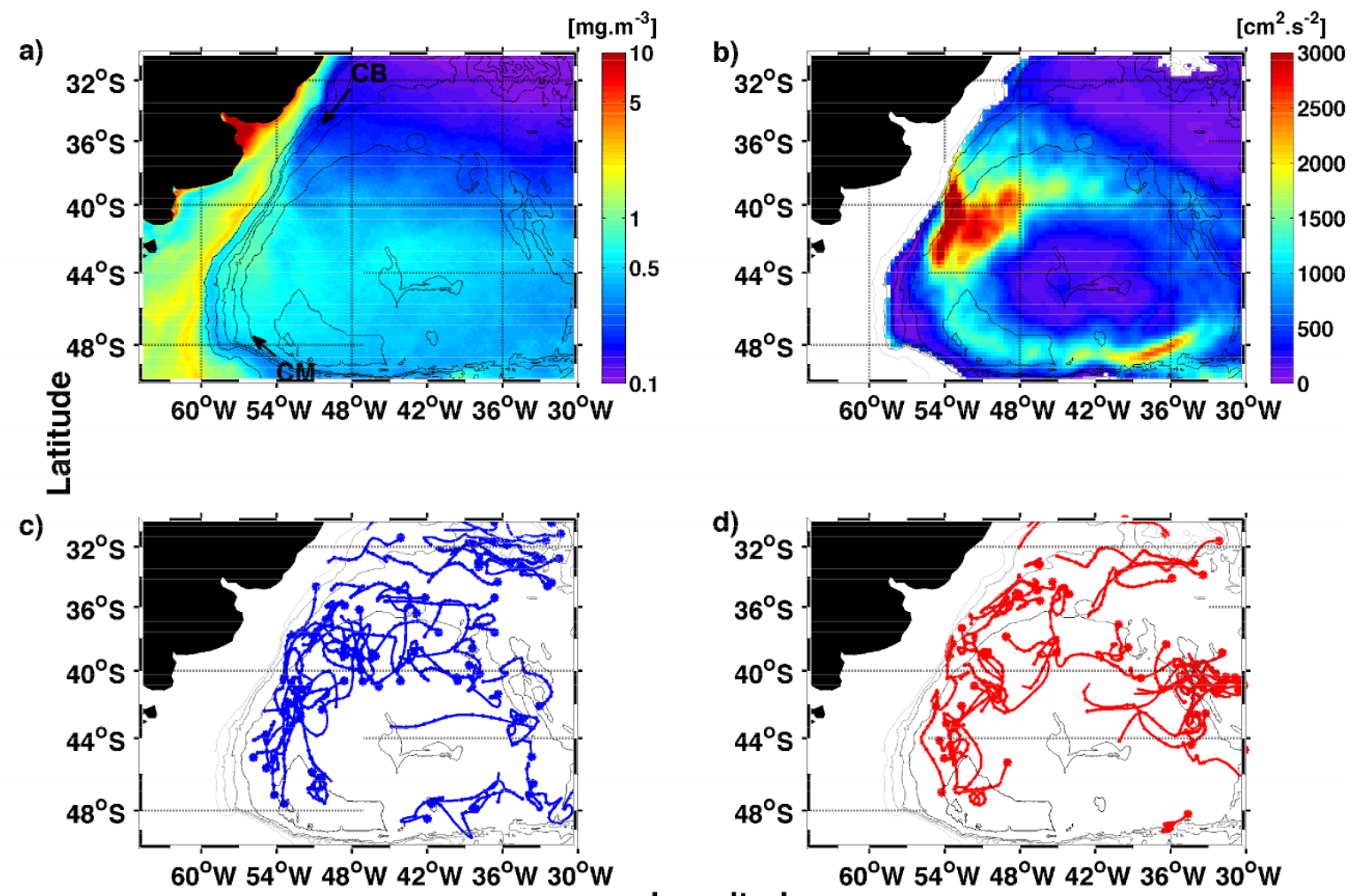

\section{Longitude}

Figura 2 - a) Concentração de clorofila média (2002 a 2010). As setas correspondem às Correntes do Brasil (CB) e das Malvinas (CM); b) Média de energia cinética turbulenta entre 2002 e 2010; c) Trajetórias dos vórtices ciclônicos e d) anticiclônicos selecionados. O local de formação de cada vórtice é indicado por um asterisco e as posições nas quais foram efetivamente amostrados são indicadas com o símbolo +.

\section{Dados de [chla]}

Foram utilizadas imagens diárias globais de nível L3 de [chla], com 9 km de resolução espacial, estimada pela aplicação do algoritmo semi-analítico Garver-Siegel-Maritorena (Maritorena et al., 2002) em dados unificados dos sensores SeaWiFS, MODIS/ Aqua e MERIS (Maritorena \& Siegel, 2005; Maritorena et al., 2010) para o período de janeiro de 2003 a dezembro de 2010. Estas imagens são disponibilizadas pelo projeto MEASURES (Making Earth Science Data Records for Use in Research Environments). Apesar destas imagens apresentarem maior cobertura espacial em relação àquelas geradas a partir de dados de um único sensor, a presença de nuvens diminui o número de píxeis válidos em imagens diárias. Levando em consideração que a posição dos vórtices é observada em snapshots de SLA, disponíveis a cada sete dias, e que os vórtices selecionados se deslocam com uma velocidade de propagação média de $\sim 11.5 \mathrm{~km}$ dia-
1 , foram utilizados mapas médios de [chla] de 3 dias centrados no dia de observação dos vórtices, o qual permite um incremento no número de píxeis válidos, mas preservando uma sincronia espacial razoável entre as observações da posição e das propriedades biológicas dos vórtices.

Finalmente, mapas de anomalias espaciais de [chla] ( $\Delta$ [chla]) foram obtidos pelo cálculo da diferença entre as imagens originais e as suavizadas pela aplicação de um filtro passa-baixa (média móvel) após a transformação logarítmica da [chla] (Siegel et al., 2011). O objetivo da aplicação do filtro passa-baixa no campo de [chla] é a remoção das assinaturas dos processos de mesoescala e sub-mesoescala, mas preservando as feições de larga escala. Desta forma os mapas filtrados servem como referência de um estado "não-perturbado" pelos processos associados aos vórtices. Após testes de sensibilidade, foi encontrado que o tamanho ótimo da janela do filtro é de $30 \times 30$ 
píxeis, equivalente a $270 \times 270 \mathrm{~km}$. Esta janela remove as assinaturas dos vórtices observados (raio 100 km) de maneira eficiente, preservando o complexo padrão de distribuição de [chla] característico da região.

\section{Esquema de amostragem}

A metodologia utilizada baseia-se naquela proposta por Siegel et al. (2011). As características físicas, bio-óticas e ambientais associadas à cada vórtice são obtidas utilizando um esquema de amostragem baseado na sua posição geográfica. Para cada um dos mecanismos físicos analisados (bombeamento vertical, bombeamento vertical de Ekman e advecção vortical de gradientes) foram obtidas variáveis que indicam a presença ou ausência do mecanismo e sua intensidade. Posteriormente, análises de correlação entre estas variáveis permitem identificar os mecanismos responsáveis pelos padrões encontrados.

A intensidade do bombeamento vortical e do bombeamento vortical de Ekman depende da amplitude do vórtice e do estresse do vento, respectivamente. O padrão espacial associado a esses mecanismos é representado pela diferença entre $\Delta[\mathrm{chla}]$ no interior e no exterior do vórtice.

A intensidade do mecanismo de advecção vortical de gradientes depende da magnitude do gradiente horizontal de [chla] e da velocidade rotacional do vórtice. O padrão espacial associado a esse mecanismo na presença de um gradiente meridional de [chla] é representado pela diferença entre a $\Delta$ [chla] nos hemisférios oeste e leste do vórtice.

Os padrões espaciais da [chla] nos vórtices selecionados foram amostrados mediante a extração dos valores de [chla] e $\Delta$ [chla] dentro de uma janela de tamanho $4 \mathrm{~L}$ x $4 \mathrm{~L}$ píxeis, onde Lé o raio instantâneo da feição ( 400 km x 400 km no caso de um vórtice com $L=100 \mathrm{~km}$ ) centrada nas posições geográficas dos vórtices. A normalização horizontal dos vórtices de acordo com o seu raio permite o cálculo de padrões médios a partir de observações de vórtices com raios variáveis (Chelton et al., 2011a). Dentro desta caixa foram selecionados os píxeis correspondentes ao interior, exterior, leste e oeste do vórtice utilizando uma máscara circular de raio correspondente ao raio instantâneo da feição (Siegel et al., 2011). Os píxeis exteriores compreendem aqueles que ocorrem na caixa de $4 \mathrm{~L} \times 4 \mathrm{~L}$ píxeis e que estão fora da área ocupada pelo raio do vórtice. Os píxeis a leste (oeste) são aqueles que ocorrem dentro da área delimitada pelo raio do vórtice, mas sendo o centro deste deslocado para a direita (esquerda) na distância equivalente ao próprio raio do vórtice.

O gradiente horizontal da [chla], indicador da distribuição horizontal da biomassa fitoplanctônica passível de modificação pela advecção vortical, foram obtidos a partir dos campos de [chla] após a aplicação do filtro passa-baixa. As componentes do vetor gradiente foram definidas como os coeficientes angulares do ajuste de uma regressão linear dentro da janela de amostragem de 4L x 4L. A Equação 1 ilustra o modelo da regressão múltipla, onde "a" é o intercepto e $\partial[\mathrm{chla}] / \partial X$ e $\partial[\mathrm{chla}] / \partial Y$ são os gradientes zonal (ao longo da longitude $\mathrm{X}$ ) e meridional (ao longo da latitude Y), respectivamente. Estes gradientes somente foram considerados quando significativamente diferentes de zero $(p<0,05)$.

$$
[\text { chla }]=a+\frac{\partial[\text { chla }]}{\partial X} X+\frac{\partial[\text { chla }]}{\partial Y} Y
$$

É importante ressaltar que na região de estudo existem também gradientes predominantemente zonais devido à proximidade com a plataforma continental, a qual se caracterizada por intensas florações de primavera (Rivas et al., 2006). Com o objetivo de obter padrões médios independentes do sentido do gradiente, previamente à extração dos dados de [chla] e $\Delta$ [chla] para cada um dos vórtices, os mapas foram rotacionados de tal forma que a direção do gradiente de [chla] local fosse para sul, de forma similar à metodologia aplicada por Chelton et al., (2011a).

O estresse do vento foi estimado a partir de dados de velocidade do vento derivados do escaterômetro Quikscat (PODAAC, 2016). Nos mapas diários, a ausência de dados é devido à limitada cobertura espacial da órbita do satélite e à contaminação dos dados devido a chuvas. Assim como no processamento dos dados de [chla], campos de vento médio de 3 dias foram calculados com o intuito de aumentar a quantidade de dados disponíveis. A velocidade do vento estimada a partir de dados de escaterômetros corresponde à velocidade relativa do vento, isto é à diferença entre a velocidade do ar e da água. Dessa maneira, o quadrado da velocidade média do vento dentro da janela de $4 \mathrm{~L}$ x $4 \mathrm{~L}$ píxeis foi utilizado como proxy do estresse do vento.

\section{RESULTADOS E DISCUSSÃO}

\section{Distribuição média da [chla] na região de estudo}

O campo médio da [chla] construído a partir de oito anos de imagens diárias (Janeiro 2003 a Dezembro de 2010, Figura 2a) é similar ao encontrado por Garcia et al. (2004) e Saraceno et al. (2005) a partir de séries de dados mais curtas do sensor SeaWiFS. Na região afastada da plataforma, local onde ocorre a maior parte dos deslocamentos dos vórtices selecionados (Figuras 2c e 2d), a [chla] aumenta para o sul. As águas oligotróficas ao norte de $40^{\circ} \mathrm{S}$ correspondem 
às águas da província biogeográfica do giro subtropical do Atlântico Sul (Longhurst, 1998). As águas ao sul de $40^{\circ} \mathrm{S}$ apresentam maior [chla] durante todas as estações do ano e correspondem à província biogeográfica da Zona de Convergência Subtropical Sul (Longhurst, 1998). Observa-se também o gradiente entre águas do oceano aberto e da plataforma continental, onde a plataforma exibe altas [chla] na imagem média devido, principalmente, às intensas florações de primavera (Rivas et al., 2006). O máximo local de [chla] associado à CBM não é visível neste campo médio, mas sim em imagens mensais, semanais (não mostrado) e diárias (Barré et al., 2006).

\section{Características dos vórtices selecionados}

Um total de 108 vórtices foram analisados, dos quais 60 são ciclônicos (Figura 2c) e 48 anticiclônicos (Figura 2d). Nenhum dos vórtices estudados propagase além da Bacia Argentina. A velocidade de translação média desses vórtices é de 11,66 km d-1 (Tabela 1), semelhante à estimada por Garcia et al. (2004) para três vórtices ciclônicos a partir de imagens de cor do oceano $\left(12 \mathrm{~km} \mathrm{~d}^{-1}\right)$. Os vórtices aqui analisados apresentam um raio médio de $102 \mathrm{~km}$, valor superior ao raio médio de $55 \mathrm{~km}$ estimado para os vórtices da CBM no estudo de Lentini et al. (2006). Tal diferença é esperada, uma vez que selecionamos vórtices de maiores raios formados na região da CBM. Vórtices anticiclônicos são maiores, propagam-se mais rápido e por maiores distâncias do que vórtices ciclônicos. Vórtices ciclônicos, por sua vez, apresentam maiores amplitudes e velocidades rotacionais, e são menos dispersivos do que vórtices anticiclônicos (i.e. maior parâmetro de não-linearidade).

Tabela 1 - Características médias (desvio padrão) dos vórtices analisados. As diferenças $\Delta[\text { chla }]_{\text {interno-externo }}$ e $\Delta[\text { chla }]_{\text {oeste-leste }}$ significativas em nível de $95 \%(p<0.05)$ estão em negrito.

\begin{tabular}{|c|c|c|}
\hline & Ciclônicos & Anticiclônicos \\
\hline Número de vórtices analisados & 60 & 48 \\
\hline Tempo de vida (semanas) & $25,45(8,52)$ & $24,75(8,15)$ \\
\hline Distância de propagação (km) & $1797,78(466,54)$ & $1947,58(289,76)$ \\
\hline Velocidade de propagação $\left(\mathrm{km} \mathrm{d}^{-1}\right)$ & $11,01(4,31)$ & $12,35(4,03)$ \\
\hline Parâmetro de não-linearidade & $3,41(2,24)$ & $2,83(1,24)$ \\
\hline Número de observações individuais & 903 & 645 \\
\hline Raio $(\mathrm{km})$ & $99,68(26,31)$ & $104,58(26,31)$ \\
\hline Amplitude (cm) & $-22,00(15,12)$ & $18,52(11,06)$ \\
\hline Velocidade Rotacional $\left(\mathrm{cm} \mathrm{s}^{-1}\right)$ & $36,68(18,92)$ & $32,71(14,06)$ \\
\hline $\begin{array}{l}\text { Idade do vórtice no momento da } \\
\text { observação (semanas) }\end{array}$ & $13,42(9,13)$ & $14,65(9,00)$ \\
\hline Velocidade do vento $\left(\mathrm{km} \mathrm{h}^{-1}\right)$ & $09,48(0,62)$ & $09,54(0,69)$ \\
\hline [chla] $\left(\mathrm{mg} \mathrm{m}^{-3}\right)$ & $0,423(0,33)$ & $0,296(0,24)$ \\
\hline$\Delta[\text { chla }]_{\text {int-ext }}\left(\log _{10} \mathrm{mg} \mathrm{m}^{-3}\right)$ & $0,027(0,05)$ & $-0,036(0,05)$ \\
\hline \multicolumn{3}{|c|}{ Vórtices em locais com gradiente [chla] para sul } \\
\hline Número de observações individuais & 542 & 389 \\
\hline $\begin{array}{l}\text { Magnitude do gradiente espacial de } \\
\text { [chla] }\left(\mathrm{mg} \mathrm{m}^{-3} \text { graus }^{-1}\right)\end{array}$ & $1,104(1,06)$ & $1,112(1,06)$ \\
\hline$\Delta[\text { chla }]_{\text {oeste-leste }}\left(\log _{10} \mathrm{mg} \mathrm{m}^{-3}\right)$ & $0,00(0,056)$ & $-0,015(0,05)$ \\
\hline \multicolumn{3}{|c|}{ Vórtices em locais com gradiente para oeste } \\
\hline Número de observações individuais & 174 & 139 \\
\hline $\begin{array}{l}\text { Magnitude do gradiente espacial de } \\
\text { [chla] }\left(\mathrm{mg} \mathrm{m}^{-3} \text { graus }^{-1}\right)\end{array}$ & $1,172(1,21)$ & $1,25(1,22)$ \\
\hline$\Delta[\text { chla }]_{\text {norte-sul }}\left(\log _{10} \mathrm{mg} \mathrm{m}^{-3}\right)$ & $0,010(0,075)$ & $0,007(0,10)$ \\
\hline
\end{tabular}

\section{Padrões espaciais da [chla] e mecanismos físicos associados}

A Figura 3 representa os padrões espaciais médios de [chla] e $\Delta$ [chla] calculados a partir de todos os vórtices ciclônicos (Figura 3a e 3c) e anticiclônicos analisados (Figura 3b e 3d). De modo geral, os padrões são consistentes com o esperado pela ação do mecanismo de bombeamento vortical, com alta [chla] e anomalias positivas associadas ao interior dos vórtices ciclônicos e baixa [chla] e anomalias negativas no interior dos vórtices anticiclônicos.

A influência dos vórtices de mesoescala pode ser melhor distinguida utilizando campos de $\Delta$ [chla] (Figura 3c e $3 d$ ) nos quais os sinais de processos de menores escalas são ressaltados. A presença de anomalias positivas associadas ao núcleo dos vórtices ciclônicos e de anomalias negativas nos anticiclônicos é evidente. A magnitude de tais anomalias é similar para ambos tipos de feição $\left( \pm 0.06 \log _{10}\right.$ [chla]). $\mathrm{Na}$ região adjacente aos vórtices são observadas anomalias de sinal oposto, mas de menor magnitude, sendo negativas para os ciclônicos $\left(-0.01 \log _{10}\right.$ [chla]) e positivas para os anticiclônicos $\left(0.03 \log _{10}\right.$ [chla]). A média das $\Delta[\text { chla }]_{\text {interna-externa }}$ (Tabela 1) confirma que a [chla] dentro dos vórtices ciclônicos é maior do que na região adjacente sendo a diferença de $0.027 \log _{10}$ [chla]. A diferença é negativa e de maior magnitude para os vórtices anticiclônicos $\left(-0.036 \log _{10}\right.$ [chla]).

\section{Bombeamento vortical}

A amplitude do vórtice é definida como a anomalia do nível do mar no centro do vórtice em relação ao nível do mar médio, sendo positiva para os vórtices anticiclônicos e negativa para os ciclônicos. A magnitude da amplitude do vórtice é diretamente proporcional à magnitude do deslocamento vertical das isopicnais. Assim, a ação do mecanismo clássico de bombeamento vortical é representada por correlações inversas significativas entre a amplitude do vórtice e a $\Delta$ [chla]. No caso de vórtices ciclônicos, menores amplitudes (mais negativas) indicam feições cujas isopicnais apresentam maiores deslocamentos, estando estas mais afastadas da sua posição de equilíbrio. Consequentemente, quanto menores as amplitudes, maior é o efeito do bombeamento vortical nos vórtices ciclônicos, devido ao maior transporte vertical de nutrientes ou de células fitoplanctônicas em direção à camada superficial. Similarmente, vórtices anticiclônicos com maiores amplitudes, estariam associados com [chla] mais baixas, devido a um maior deslocamento das isopicnais em direção ao fundo. As correlações inversas esperadas foram encontradas tanto para vórtices ciclônicos quanto anticiclônicos, sendo os coeficientes de correlação parcial significativos e iguais a $-0,28$ e $-0,20$, respectivamente. Essa correlação entre 


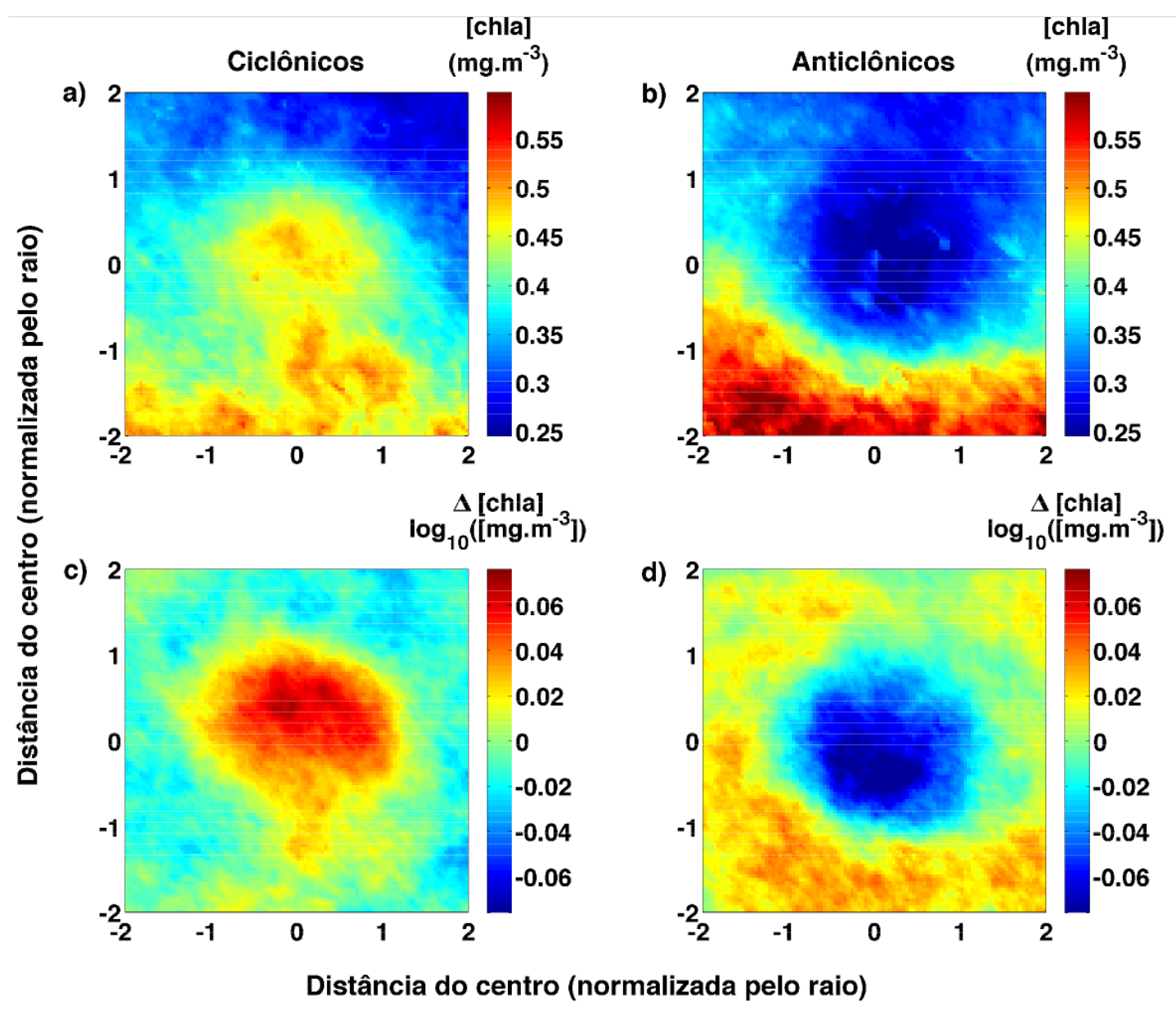

Figura 3 - Campos médios de [chla] (a e b) e de $\Delta$ [chla] (c e d) de todas as observações dos vórtices ciclônicos (direita) e anticlônicos (esquerda). Os campos de [chla] e $\Delta$ [chla] de cada vórtice observado foram rotacionados a fim de se obter gradientes sempre aumentando para sul.

amplitudes dos vórtices e $\Delta$ [chla] evidencia a ação do mecanismo de bombeamento vortical. Avaliando os mesmos parâmetros utilizados neste trabalho, Siegel et al. (2011) encontraram um coeficiente de correlação de -0.53 para vórtices ciclônicos no Mar dos Sargaços, consideravelmente maior ao encontrado para a CBM $(r=-0.28)$. Por outro lado a correlação entre a anomalia de [chla] e a amplitude dos vórtices anticiclônicos não foi significativa no Mar dos Sargaços (Siegel et al., 2011). Tais diferenças são esperadas devido as características biogeoquímicas diferenciadas em ambas as regiões. O Mar dos Sargaços é predominantemente oligotrófico, sendo que as águas acima da picnoclina são praticamente esgotadas em nutrientes. Neste local, os vórtices ciclônicos têm sido sugeridos como uma importante fonte de nitrogênio para a produção primária nova (McGillicuddy et al., 2007). Ainda, o enfraquecimento de vórtices anticiclônicos nesta região causaria deslocamento das isopicnais em direção ao seu estado de equilíbrio, porém não disponibilizaria águas ricas em nutrientes para a zona eufótica. Por sua vez, a produção primária na região da CBM ao sul de $37^{\circ} \mathrm{S}$, depende do ciclo sazonal de estratificação da coluna da água (Rivas e Piola, 2002), uma vez que a advecção de águas do Rio da Prata em direção ao oceano aberto (Brandhorst e Castello, 1971), assim como o fluxo da $\mathrm{CM}$, garantem a disponibilidade de nutrientes. Desta forma, a importância relativa da injeção de nutrientes causada pelos vórtices na CBM é menor que a do seu papel auxiliando os processos de mistura e estratificação. Ainda, o enfraquecimento dos vórtices anticiclônicos nesta região pode resultar em uma ressurgência de águas mais ricas em nutrientes, de forma similar ao mecanismo descrito para os Haida Eddies do Golfo do Alaska (Crawford et al., 2005), explicando a correlação encontrada.

\section{Bombeamento vortical de Ekman}

Uma correlação inversa significativa, mas de pequena magnitude, entre o estresse do vento e a $\Delta[\text { chla }]_{\text {interna-externa }}(r=-0.04)$ evidencia a ação deste mecanismo em vórtices ciclônicos. Esta correlação é esperada, pois a assimetria espacial no estresse do vento causa convergência do transporte de Ekman 
no interior de vórtices ciclônicos, removendo células fitoplanctônicas da camada superficial e resultando em uma diminuição na [chla].

Por outro lado, este mecanismo não foi evidenciado no caso dos vórtices anticiclônicos analisados neste estudo. A ressurgência associada ao bombeamento vortical de Ekman resultaria em um aumento da [chla] devido à injeção de nutrientes. Tal mecanismo foi inicialmente proposto para vórtices de água modal do Mar dos Sargaços (Martin \& Richards, 2001), os quais se caracterizam por uma estrutura vertical diferenciada: como nos demais vórtices anticiclônicos, a picnoclina permanente encontra-se deslocada em direção ao fundo, mas a sua picnoclina sazonal encontra-se deslocada em direção à superfície, resultando em uma resposta biológica similar à dos vórtices ciclônicos. Tal estrutura implica em uma maior disponibilidade de nutrientes para serem transportados em direção à superfície pelas velocidades verticais decorrentes do bombeamento vortical de Ekman. Vórtices de água modal não foram descritos no Atlântico Sudeste, justificando a ausência da uma ressurgência causada por bombeamento vortical de Ekman em vórtices anticiclônicos da região.

\section{Advecção vortical de gradientes}

Na região de oceano aberto da CBM vórtices deslocam-se predominantemente de maneira zonal ao longo de um gradiente de [chla] meridional em direção ao sul. Já na região próxima ao talude continental, vórtices deslocam-se de maneira meridional ao longo de um gradiente de [chla] zonal em direção ao oeste. Esse gradiente para oeste se dá devido ao contraste de [chla] entre a plataforma continental e o oceano aberto. Cerca de $60 \%$ das observações dos vórtices ocorreram em locais com gradientes para sul e $20 \%$ em locais para oeste. $O$ valor médio do gradiente é de $1,1 \mathrm{mg} \mathrm{m}^{-3}$ graus $^{-1}$ para sul e de $1,2 \mathrm{mg} \mathrm{m}^{-3}$ graus $^{-1}$ para oeste. Em ambos os casos os desvios da média são da mesma magnitude, indicando grande variabilidade.

Esta distribuição bimodal dos gradientes de [chla] foi removida rotacionando os mapas de [chla] de tal forma que a direção do gradiente local seja para sul. Desta forma o padrão médio da Figura 3 evidenciaria o efeito da advecção vortical independentemente da direção do gradiente. Ao se deslocarem por uma região cuja [chla] aumenta em direção ao sul, a velocidade rotacional dos vórtices advectaria parcelas de água com altas [chla] para norte no lado oeste (leste) dos vórtices ciclônicos (anticiclônicos). Ao mesmo tempo, águas com baixas [chla] seriam advectadas para sul no lado leste (oeste) dos vórtices ciclônicos (anticiclônicos). Tal distorção do gradiente de [chla] de acordo com o sentido do giro dos vórtices não é aparente nos padrões espaciais médios da Figura 3.
Ao invés deste padrão assimétrico, observa-se que em ambos os hemisférios dos vórtices ciclônicos (anticiclônicos) tanto a [chla] quanto a $\Delta$ [chla] é menor (maior) que no centro.

A diferença entre os hemisférios dos vórtices que ocorreram em regiões com gradiente de [chla] para sul $\left(\Delta[\text { chla }]_{\text {oeste-leste }}\right)$ foi negativa $\left(-0.015 \log _{10}\right.$ [chla]) para feições anticiclônicas, como esperado pela ação da advecção vortical. No caso de vórtices anticiclônicos que se deslocaram por regiões com gradientes para oeste, a diferença média $\Delta[\mathrm{chla}]_{\text {norte-sul }}$ apesar de ser significativa foi próxima de zero $(0,007$ $\log _{10}$ [chla]) e apresentou uma considerável variabilidade, com um desvio padrão de $0,1 \log _{10}$ [chla]. Os vórtices ciclônicos, independentemente da direção do gradiente de [chla], não apresentaram uma diferença entre os seus hemisférios que fosse significativamente diferente de zero.

Teoricamente, a magnitude da diferença entre os hemisférios $\Delta[\mathrm{chla}]_{\text {oeste-leste }}$ dos vórtices anticiclônicos com gradiente de [chla] para sul é função da intensidade do próprio gradiente de [chla] e da velocidade rotacional do vórtice. A correlação positiva entre $\Delta[\mathrm{chla}]_{\text {oeste-leste }}$ e a magnitude da velocidade rotacional foi encontrada $(r=0.16)$ indicando que as propriedades físicas dos vórtices determinam a magnitude da distorção dos gradientes devido à advecção vortical.

Chelton et al. (2011a) demonstraram que o mecanismo de advecção vortical de gradientes é responsável pelo padrão de propagação para oeste observado nas anomalias da [chla] superficial. Esse padrão foi anteriormente atribuído à ação de ondas de Rossby não-lineares. A evidência da ação desse mecanismo na CBM sugere que a propagação para oeste de duas anomalias de [chla] na CBM previamente atribuídas à ondas de Rossby intrasazonais (Garcia et al., 2004), pode ser resultado da advecção vortical de vórtices de mesoescala.

\section{Advecção horizontal de fluído}

Todos os vórtices estudados, exceto um ciclônico, apresentaram comportamento não-linear. Isto indica que a advecção horizontal de fluído pode ser um mecanismo ativo nas assinaturas de alta (baixa) [chla] em vórtices ciclônicos (anticiclônicos). Dessa maneira, as assinaturas de [chla] da Figura 3 sugerem que vórtices ciclônicos (anticiclônicos) formados em uma região de alta [chla] se propagam para uma região de baixa [chla] mas ainda retém a assinatura de [chla] de sua região de formação. Logo, o efeito do parâmetro de não-linearidade na assinatura de [chla] do vórtice depende das condições biológicas prévias do local da formação do vórtice. Para investigar esse mecanismo, seria necessário analisar a [chla] dos vórtices durante sua formação (i.e. previamente à sua identificação 
como uma feição de linhas de corrente fechadas). Esse período não está incluído na base de dados aqui utilizada.Contudo, a existência de uma relação entre a idade do vórtice no momento da observação e a $\Delta[\mathrm{chla}]_{\text {interna-externa }}$ pode ser interpretada como um indício da ação da adevecção horizontal de fluído. A Figura 4 mostra a evolução temporal da $\Delta[\text { chla }]_{\text {interna-externa }}$ para os vórtices analisados. Para ambas as polaridades, a $\Delta[\mathrm{chla}]_{\text {interna-externa }}$ tem maior magnitude logo após a formação do vórtice e decresce com o tempo. Tal padrão indica que a advecção horizontal de fluido tem um papel na determinação das assinaturas observadas, pois a capacidade de advecção não-linear dos vórtices decai com o tempo. Isto dá lugar à mistura das águas do interior do vórtice com as águas exteriores, resultando em menores anomalias dentro dos vórtices ao longo do seu tempo de vida.

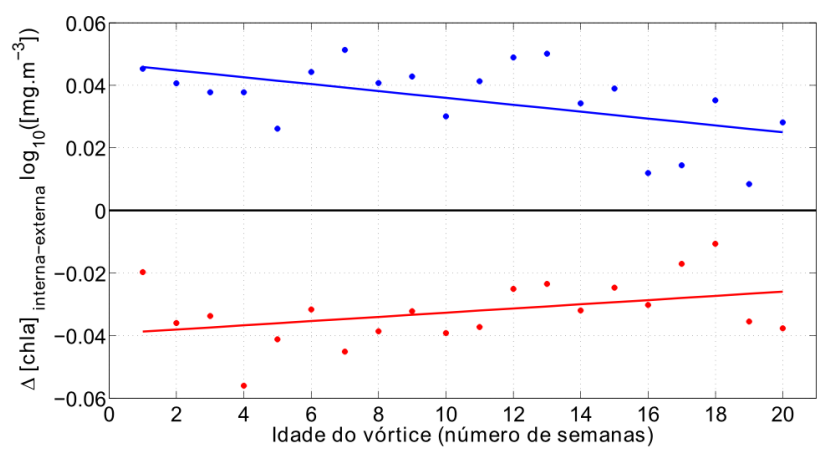

Figura 4 - Evolução da diferença $\Delta$ [chla]interna-externa em vórtices ciclônicos (azul) e anticlônicos (vermelho) ao longo do seu tempo de vida. Os pontos correspondem aos valores médios e as linhas às regressões ajustadas, sendo a idade do vórtice a variável independente. A inclinação da reta é de -0.0011 e $0.0007 \log _{10} \mathrm{mg} \mathrm{m}^{-3}$ semana-1 para vórtices ciclônicos e anticiclônicos, respectivamente. As regressões foram significativas ao nível de $95 \%$ e os valores do $\mathrm{R}^{2}$ foram de 0.27 (ciclônicos) e 0.15 (anticiclônicos).

\section{Assinatura dos vórtices na [chla]: combinação de mecanismos}

A ação do mecanismo clássico de bombeamento vortical nos padrões espaciais médios da $\Delta$ [chla] (Figura 3c e 3d) parece evidente, uma vez que anomalias positivas estão associadas ao interior dos vórtices ciclônicos e anomalias negativas ocorrem nos vórtices anticiclônicos. No entanto, este padrão médio resulta da somatória dos efeitos do bombeamento vortical, do bombeamento vortical de Ekman, e da advecção não-linear de fluído dentro do vórtice. Logo, o padrão observado na Figura 3c e $3 d$ é produto de uma combinação de mecanismos.

O mecanismo de bombeamento vortical, ocorre principalmente durante a formação dos vórtices e durante a sua interação com outras feições. Esse mecanismo transporta nutrientes e/ou células de fitoplâncton verticalmente e confere aos vórtices características físicas e biogeoquímicas distintas daquelas presentes nas águas adjacentes. A advecção horizontal de fluído tem maior magnitude após a formação do vórtice, decaindo com o tempo. Esse mecanismo permite que as águas no interior dessas feições sejam capazes de conservar suas características diferenciadas ao longo da sua trajetória, auxiliando então no transporte de energia e massa nos oceanos (e.g. Olson, 1991). Dessa maneira, a advecção horizontal do fluído auxilia na manutenção do padrão original de [chla] causado pelo bombeamento do vórtice.

A CB retroflexiona aproximadamente aos $38^{\circ} \mathrm{S}$, devido à inclinação da costa em sentido oeste em relação ao fluxo da corrente (Olson et al., 1988). A oclusão do primeiro meando dessa retroflexão dá origem a vórtices de mesoescala. Dessa maneira, a formação destas feições é geograficamente controlada (Garcia et al., 2004). Na região da retroflexão da CB há um forte gradiente meridional de propriedades físicas (temperatura e salinidade) e biogeoquímicas (nutrientes e [chla]), devido ao encontro de diferentes massas de água transportadas pela $\mathrm{CB}$ e pela $\mathrm{CM}$. Portanto, a propagação de feições de mesoescala coerentes (não-lineares) formadas na CBM, juntamente com o fluxo anticiclônico Zapiola, auxiliaria a mistura das águas através da frente (Garzoli et al., 2008). No entanto, as trajetórias dos vórtices estudados indicam que estas estruturas auxiliam no transporte de águas nas mais variadas direções e não somente através da frente.

O padrão espacial médio da $\Delta$ [chla] associado ao vórtices (Figura 3c e 3d) confirma que a propagação destes pode ser observada em imagens da cor do oceano como uma anomalia positiva ou negativa dependendo da polaridade da feição. Uma vez apresentando comportamentos não-lineares, essas feições transportariam águas mais ou menos ricas em biomassa e nutrientes, desempenhando assim um papel importante na determinação da distribuição espacial da [chla] na área de estudo. Este resultado suporta o mecanismo proposto por Saraceno et al. (2005) de que a alta [chla] observada durante a primavera e o verão na região do overshoot da CB seria mantida pelo aporte de águas ricas realizado pelos vórtices.

A impermeabilidade dos vórtices, associada ao seu comportamento não-linear, é somente uma aproximação teórica que não considera a variabilidade temporal da dinâmica dos vórtices (Lenhan et al., 2007). Outros fatores como as características das estruturas lagrangianas coerentes ao redor do vórtices são importantes na sua determinação (Lehahn et al., 2011). Dessa maneira, estudos de rastreamento de partículas inicialmente presas em vórtices, como o realizado por Lehahn et al. (2011), são essenciais para investigar a capacidade de transporte horizontal dos vórtices da CBM. Tais estudos auxiliariam no discernimento 
da importância biogeoquímica de vórtices na região.

Ainda um outro mecanismo que influencia a [chla] associada a vórtices é a presença de filamentos (Lévy et al., 2001; Calil e Richards, 2010). Calil e Richards (2010), por exemplo, observaram filamentos de altas [chla] ao redor de vórtices de mesoescala próximo às ilhas do Havaí, e os atribuíram a processos de frontogênese resultado da intensificação de frentes de temperatura pelo campo de velocidade vortical. A ação deste mecanismo não foi avaliada neste trabalho, mas a presença de anomalias positivas ao redor dos vórtices anticiclônicos pode ser interpretada como indicio da sua atividade na CBM. A escala espacial de processos de submesoescala, assim como o dinamismo da sua localização geográfica, dificulta a sua visualização nos padrões médios de [chla] (Figura 3). A metodologia utilizada neste trabalho não é apropriada para o estudo de tais processos, os quais requerem a análise de imagens instantâneas de [chla].

\section{CONSIDERAÇÕES FINAIS}

A metodologia proposta por Siegel et al. (2011) foi adaptada e aplicada na parcela sudoeste do Oceano Atlântico Sul com o fim de inferir quais mecanismos contribuem na determinação do padrões espaciais de [chla] associados aos vórtices de mesoescala da região. Tanto os padrões espaciais médios da [chla] e da $\Delta$ [chla], quanto os resultados das análises de correlação indicaram que o bombeamento vortical tem grande influência de nos padrões de [chla]. Adicionalmente, o mecanismo de advecção não-linear de fluido auxilia na manutenção do padrão originado pelo mecanismo de bombeamento vortical. As assinaturas dos mecanismos de bombeamento vortical de Ekman e de advecção vortical de gradientes foram evidentes somente em alguns casos, sendo tais mecanismos secundários no estabelecimento dos padrões observados.

Estudos sobre as características dos vórtices da CBM, especificamente em relação ao seu grau de permeabilidade, capacidade de transporte e trajetórias, são necessários para discernir os efeitos dos mecanismos de bombeamento vortical e de advecção horizontal na distribuição superficial da [chla] da região. Ainda, em uma região com uma sazonalidade pronunciada, como a CBM, futuros estudos devem avaliar a variabilidade sazonal das assinaturas dos vórtices na [chla]. Além disso, os vórtices de mesoescala são estruturas tridimensionais. Logo, a utilização de dados de sensoriamento remoto, limitados à observação da superfície, apresenta restrições. Os dados da cor do oceano representam valores médios das propriedades na primeira profundidade ótica e, portanto, a [chla] estimada a partir do sensoriamento remoto não inclui a biomassa presente em toda a coluna d'água. Considerando que o impacto biogeoquímico dos vórtices é mais importante na base da camada eufótica (McGillicuddy et al., 2007), também são necessários estudos da estrutura tridimensional dos vórtices, para entender as interações físico-biológicas ao longo da coluna d'água. Dados in situ coletados em campanhas oceanográficas tradicionais e por plataformas autônomas, como flutuadores Bio-Argo e gliders, devem ser utilizados como ponto de partida e fonte de validação de experimentos de modelagem numérica orientados ao estudo dos efeitos dos mecanismos na coluna d'água.

\section{REFERÊNCIAS BIBLIOGRÁFICAS}

Arístegui, J.; et al. 1997. The influence of islandgenerated eddies on chlorophyll distribution: a study of mesoscale variation around Gran Canaria. Deep Sea Res. I: Oceanographic Research Papers, 44(1): 71-96.

Barré, N.; Provost, C.; Saraceno, M. 2006. Spatial and temporal scales of the Brazil-Malvinas Current confluence documented by simultaneous MODIS Aqua $1.1-\mathrm{km}$ resolution SST and color images. Advances in Space Research 37: 770-786.

Brandhorst, W.; Castello, J.P. 1971. Evaluación de los recursos de anchoita (Engraulis anchoita) frente a la Argentina y Uruguay I: las condiciones oceanograficas, sinopsis del conocimiento actual sobre la anchoita y el plan para su evaluación. Proyecto de Desarrollo Pesquero, Serie Informes Tecnicos, Mar del Plata 29, 1-63.

Brandini, F.P.; Boltovskoy, D.; Piola, A.; Kocmur, S.; Rottgers, R.; Abreu, P.C.; Lopes, R.M. 2000. Multiannual trends in fronts and distribution of nutrients and chlorophyll in the southwestern Atlantic (3062ㅇ). Deep Sea Res. I 47: 1015-1033.

Calil, P. H. R. \& Richards, K. J. 2010. Transient upwelling hot spots in the oligotrophic North Pacific. Journal of Geophysical Research, 115(C2), C02003-C02003. doi:10.1029/2009JC005360.

Chelton, D. B.; Gaube, P.; Schlax,M. G.; Early, J. J. \& Samelson, R. M. 2011a. The Influence of Nonlinear Mesoscale Eddies on Oceanic Chlorophyll. Science, 334: 328-332.

Chelton, D.B.; Schlax, M.G. \& Samelson, R.G. 2011b. Global observations of nonlinear mesoscale eddies. Progr. Oceanogr. 91 (2): 167-216.

Crawford, W. R.; Brickley, P. J; Peterson, T. D. \& Thomas, A. C. 2005. Impact of Haida Eddies on chlorophyll distribution in the Eastern Gulf of Alaska. Deep Sea Res. II: Topical Studies in 
Oceanography. 52(7-8): 975-989.

Crawford, W. R.; Brickley, P. J. Thomas, A. C. 2007. Mesoscale eddies dominate surface phytoplankton in northern Gulf of Alaska. Progr. Oceanogr. Volume 75(2): 287-303.

Eden, C. \& Dietze, H. 2009. Effects of mesoscale eddy/ wind interactions on biological new production and eddy kinetic energy. J. Geophys. Res. 114(C5): C05023-C05023.

Flierl, G. R. 1981. Particle motions in large-amplitude wave fields. Geophysical \& Astrophysical Fluid Dynamics. 18(1-2): 39-74.

Garcia, C.A.E.; Sarma, Y.V.B.; Mata, M.M. \& Garcia, V.M.T. 2004. Chlorophyll variability and eddies in the Brazil-Malvinas Confluence Region. Deep Sea Res. II. 51: 159-172.

Garzoli, S.L.; Piola, A.; Speich, S.; Baringer, M.; Goni, G; Donohue, K.; Meinen, C.; Matano, R.; 2008. A monitoring system for heat and mass transports in the South Atlantic as a component of the meridional overturning circulation. Tech. Rep. ICPO Publication Series no.125, Available from: eprints.soton.ac.uk/50121/01/125_SAMOC_ report_Jan08.pdf.

Lehahn, Y.; d'Ovidio, F.; Lévy, M. \& Heifetz, E. 2007. Stirring of the northeast Atlantic spring bloom: A Lagrangian analysis based on multisatellite data. J. Geophys. Res. 112(C8): C08005.

Lehahn, Y.; d'Ovidio, F.; Lévy,M.; Amitai, Y. \& Heifetz, E. 2011. Long range transport of a quasi isolated chlorophyll patch by an Agulhas ring. Geophys. Res. Lett. 38: L16610.

Lentini, C.A.D.; Goni, G.J. \& Olson, D.B. 2006. Investigation of Brazil Malvinas Current rings in the confluence region. J. Geophys. Oceanogr. 111(C6).

Lévy, M.; Klein, P.; \& Treguier, A. 2001. Impact of submesoscale physics production and subduction of phytoplankton in an oligotrophic regime. Journal of Marine Research, 535-565.doi: $10.1357 / 002224001762842181$.

Longhurst, A.1998. Ecological Geography of the Sea. Elsevier. New York.398 p.

Maritorena, S. \& Siegel, D.A. 2005. Consistent Merging of Satellite Ocean Color Data Sets Using a BioOptical Model. Remote Sens. Environ. 94(4).

Maritorena, S.; Hembise-Fantos-d'Andon, O.; Mangin, A. \& Siegel, D.A. 2010. Merged Satellite Ocean Color Data Products Using a Bio-Optical Model: Characteristics, Benefits and Issues. Remote Sens. Environ. 114(8).

Maritorena, S.; Siegel, D.A. \& Peterson, A. 2002. Optimization of a Semi-Analytical Ocean Color Model for Global Scale Applications. Applied Optics. 41(15): 2705-2714.

Martin, A.P. \& Richards, K.J. 2001. Mechanisms for vertical nutrient transport within a North Atlantic mesoscale eddy. Deep Sea Res. II-Topical Studies in Oceanography. 48(4-5): 757-773.

McGillicuddy, D. \& Robinson, A. 1997. Eddy-induced nutrient supply and new production in the Sargasso Sea. Deep Sea Res. I: Oceanographic Research Papers. 44(8). 1427-1450.

McGillicuddy, D. et al. 2007. Eddy/wind interactions stimulate extraordinary mid-ocean plankton blooms. Science. 316(5827). 1021-1026.

Olson, D. B. 1991. Rings in the Ocean. Annual Review Of Earth And Planetary Sciences. 19: 283-311

Olson, D.B.; Podesta, G.P.; Evans, R.H. \& Brown, O.B. 1988. Temporal variations in the separation of the Brazil and Malvinas Currents, Deep Sea Res., Part A, 35, 1971-1990.

PODAAC (Physical Oceanography Distributed Active Archive Center). 2013. Dados Quikscat disponibilizados em ftp://podaac-ftp.jpl.nasa. gov/allData/quikscat/L3/jpl/, acessados em Junho/2013.

Rivas, A. L.; Dogliotti, A. I. \& Gagliardini, D. A. 2006. Seasonal variability in satellite-measured surface chlorophyll in the Patagonian Shelf. Continental Shelf Res. 26: 703-720.

Rivas, A.L., Piola, A.R., 2002. Vertical stratification at the shelf off northern Patagonia. Continental Shelf Research 22, 1549-1558.

Robinson, A. (1983), Eddies in Marine Science, Springer-Verlag.

Saraceno, M.; Provost C. \& Piola, A. R. 2005. On the relationship of satellite retrieved surface temperature fronts and chlorophyll-a in the Western South Atlantic. J. Geophys. Res. 110: C11016.

Saraceno, M.; \& Provost, C. 2012. On eddy polarity distribution in the southwestern Atlantic. Deep Sea Res. I: Oceanographic Research Papers. 69: 62-69.

Siegel, D.A.; Court, D.B.; Menzies, D.W.; Peterson, A.; Maritorena, S. \& Nelson, N.B. 2008. Satellite and in situ observations of the bio-optical signatures of two mesoscale eddies in the Sargasso Sea. Deep Sea Res. II. 55: 1218-1230.

Siegel, D.A.; Peterson,P.; McGillicuddy, J.R.; Maritorena, S. \& Nelson, N.B. 2011. Bio-optical footprints created by mesoscale eddies in the Sargasso Sea. Geophys. Res. Let. 38: L13608.

Silveira, I.C. A.; Schimdt, A.C.K.; Campos, E.J.D.; Godoi, S.S. \& Ikeda, Y. 2000. A Corrente do Brasil ao Largo da Costa Leste Brasileira. Revista Brasileira de Oceanografia. 48:171-18.

Souza, R. B.; Mata, M. M.; Garcia, C. A. E.; Kampel, M.; Oliveira, E. N.; Lorenzzetti, J. A. 2006. Multisensor satellite and in situ measurements of a warm core ocean eddy south of the Brazil-Malvinas 
Angel-Benavides, I.M., et al. (2016). Influência de vórtices na concentração de clorofila da CBM.

Confluence region. Remote Sens. Environ. 100(1): 52-66.

Tilburg, C. E., B. Subrahmanyam, \& O'Brien, J. J. 2002. Ocean color variability in the Tasman Sea.

Geophys. Res. Let. 29(10): 1487.

Submetido: Agosto/2013

Revisado: Outubro/2015

Aceito: Janeiro/2016 